\title{
Observations of nonmagnetic CP stars: Crossing boundaries
}

\author{
Glenn M. Wahlgren \\ Lund Observatory, University of Lund, Box 43, SE-22100 Lund, Sweden \\ email: glenn.wahlgren@astro.lu.se
}

\begin{abstract}
Observations of chemically peculiar (CP) stars have been conducted for decades and have revealed a variety of spectrum anomalies, most prominent among them are line enhancements of heavy elements. The earlier observations were limited to the optical region and the use of less sensitive detectors, yet are responsible for much of our current characterization of the $\mathrm{CP}$ star phenomenon. More recent observations embrace a wider expanse of the electromagnetic spectrum and employ more sensitive detectors that continue to unveil new levels of spectrum peculiarity. The traditional criteria used to distinguish normal from peculiar stars have become blurred, thus in some sense replacing the concept of peculiarity with one of continuity. This presentation will address the observations of the traditional nonmagnetic CP star groups over the past decade, paying particular attention to new avenues of research that have a bearing upon the interpretation of the atmospheres of CP stars and the origins of this phenomenon.
\end{abstract}

Keywords. Stars: abundances, stars: chemically peculiar, stars: magnetic fields

\section{Introduction}

Traditionally, the CP stars of the upper main sequence extend from spectral type mid$\mathrm{B}$ at the hotter end through the A type stars and into spectral type F. The inclusion of the roAp stars now extends this range to essentially the start of spectral type G. Traditionally, there are two parallel tracks of stars considered, one magnetic and the other nonmagnetic, with the nonmagnetic chemically peculiar (nmCP) stars being comprised of the HgMn, hot-Am, Am and Fm stars. The case of the He-weak stars is particularly troubling in that many of them display magnetic signatures, thereby making them interlopers between the He-strong and Bp stars. Other He-weak stars have spectral signatures that make them hotter analogs of the HgMn stars. As a class, designated mostly by the nonunique characteristic of a helium deficiency in the observable atmosphere, the He-weak stars serve as a buffer zone between the magnetic and nonmagnetic tracks. Traditionally, we have used low and medium spectral resolution data and have been blind to the plethora of peculiarity that pervades this spectral region. As essentially no star in this region is chemically normal (displaying solar-like chemical composition) the boundaries imposed by classification schemes are becoming blurred by discoveries made from high resolution spectra of peculiarities that appear to cross boundaries.

Much progress has been made in this field during the past decade. What can be consider to be the last thorough review of B and A star properties occurred in 1992, at IAU Colloquium No. 138. Subsequent conferences related to stars of these spectral types were for the most part dedicated to the subject of magnetic fields, often with solitary contributions to peripheral topics. It was also during the early to mid 1990s that one starts to see benefits from the changes in observation techniques from using photographic emulsions to a more prominent role of the silicon diode arrays and CCDs. 
This quantum leap in detector sensitivity allowed for more subtle details of the spectrum to be recorded, and subsequently encouraged new areas of stellar astrophysics.

From these conferences and the published journal literature one decade ago the topics under study can be categorized as follows: basic properties (colors, fluxes, temperatures, classification), binarity (statistics), spectrum analysis (elemental abundances, isotopic composition, the extension into the UV from HST observations, and rumblings about stratification), magnetic fields (presence in nmCP stars), variability (both photometric and spectroscopic), and new wavelength regimes for observations (x-ray, radio). Research has matured in each of these areas over the past decade and is essentially the content of this present review, with emphasis placed on spectroscopic analyses. It is not the purpose of this discussion to be complete in its bibliography, but rather to present examples that have advanced our knowledge and display the wealth of new phenomena that need to be considered to provide a complete picture of the atmospheres of nmCP stars.

\section{Binarity}

As a result of improvements in the quality of spectra, companions to HgMn stars continue to be identified, and we should expect to find additional cases of binarity among currently known Am and HgMn stars (see, e.g., Iliev et al. 2005) Most literature still cites older statistics that claim binarity among HgMn stars to be no more prevalent than among the general field stars, which is to say about $50 \%$. However, this conflicts with published statements (Hubrig \& Mathys 1995, Mathys \& Hubrig 1995) claiming a lower limit perhaps as high as $67 \%$ and the general impression that binarity is more prevalent since companions continue to be discovered. A revision in this number is needed to perhaps clear up any misconceptions. What is known is that HgMn stars have a high presence among SB2 systems and the number of known triple systems is also increasing. Am stars have a high presence in binary systems. Further it has been suggested that HgMn star companions with $T_{\text {eff }}<10000 \mathrm{~K}$ are Am stars (Ryabchikova 1998).

The study of main sequence B and A star companions to Cepheid variables (Evans 1995) has detected CP phenomena in several of these companions. This work has implications for the onset of CP star characteristics through evolutionary dating of the Cepheid variable. An unexpected result of this work has been the detection of a third star as the close companion to the main sequence B star. To date, the systems SU Cyg (HgMn companion, Wahlgren \& Evans 1998), T Mon (Bp companion, Evans et al. 1999) and AW Per (B8 V companion, Evans et al. 2000) have been studied. Although there is still uncertainty in the CP star nature of the companion to AW Per, it is interesting to consider that each proported CP star is accompanied by a close companion.

A final note on the issue of binarity regards the early detection of X-rays in late B type stars by the EINSTEIN and the ROSAT satellites. Weak X-ray emission from several late B type stars, including HgMn stars, was suggested to be the result of wind processes. Subsequent X-ray observations obtained at higher spatial resolution from the CHANDRA satellite observatory and ground based IR imaging have detected red companions to these binary systems (Stelzer et al. 2003), in agreement with the idea of an active late-type companion. The idea of wind induced X-rays in late B-type stars seems now to be a less likely explanation for observed emission.

\section{Elemental abundances}

Much work has been undertaken in the discipline of elemental abundance determination for $\mathrm{nmCP}$ stars. However, the nature of the work has not been the same for the $\mathrm{HgMn}$ 
stars as for the Am stars. For the Am stars the majority of studies published during the past decade address members in stellar clusters with the underlying purpose being to determine the onset of peculiarity, its development with cluster age, the frequency of $\mathrm{CP}$ stars in clusters and to provide tests for diffusion theory (see, e.g., Burkhart \& Coupry 1997, Hui-Bon-Hoa et al. 1997, Hui-Bon-Hoa \& Alecian 1998, Savanov 1998). This line of inquiry is more natural for the Am than HgMn stars since the latter are found to be a more rare occurrence in open clusters as a result of their faster pace for evolution. The work on cluster Am stars is by no means complete, as can be seen from presentations at this conference (Monier \& Richard, 2005).

The Am star elemental abundance work is being conducted using optical region spectra, which means that the basic picture of the abundance pattern remains the same as it has been for the past several decades. Although there exists a range for abundances of individual elements, in general, a trend exists for increasing abundance with atomic number (Smith 1996) with the enhancements reaching up to two orders of magnitude. The sampling of the periodic table is rather poor for elements heavier than the iron group, with the exception of the lanthanides. High quality ultraviolet data are needed to extend these analyses to elements that may best be detected through ultraviolet transitions. Unfortunately, the low resolution of the IUE satellite spectra and the extremely limited data obtained from the $H S T$ for Am stars will not serve to improve this situation. The importance of filling-in the existing abundance pattern lies in following the trends in abundance to both higher and lower temperatures, i.e. to cross boundaries of classification, and in providing theoreticians with additional constraints for modeling processes of atmospheric dynamics.

In contrast to the Am stars, the abundance analyses for HgMn stars tend to focus on stars as individuals or address specific tasks (isotopic composition) from observations of a group of stars. In this way progress has been made on including additional elements to better define the abundance pattern and in identifying new atmospheric phenomena from the spectra. The ultraviolet spectral region has played a key role in defining the elemental abundance distributions of HgMn stars. IUE spectra have been collected for a number of HgMn stars and studied with synthetic spectrum techniques for specific elements. A series of papers by K.C.Smith (e.g., 1995, 1997) address abundances for several elements and searches for trends of abundance with temperature. Similar analyses from coadded IUE spectra have also been undertaken by Adelman et al. (1993), Wahlgren et al. (1993) and Adelman et al. (2004) for the heaviest elements. Using optical wavelength data Adelman and collaborators have continued to analyse elemental abundances for bright HgMn, Am, and normal main-sequence B and A type stars.

The HgMn star having the highest fidelity for its elemental abundance distribution is $\chi$ Lupi. This star has been the focus of HST observations that were conducted during the first half of the $1990 \mathrm{~s}$, but continue to reveal new results as new atomic data are produced to analyse the spectrum. The $\chi$ Lupi Project (Leckrone et al. 1999, and subsequent papers on individual elements) has produced a benchmark by which other HgMn stars can be compared. The most recent results for osmium and iridium (Ivarsson et al. 2004) have lead to defining a clear abundance peak among the heaviest stable elements (Fig. 1). The peak is independent of ionization stage and its explanation will require input from theoreticians and atomic spectroscopists. It remains to be seen whether this same peak structure is reproduced by other HgMn stars, and if not, then to determine which parameters (temperature, age, etc.) are responsible for the observed peak and its evolution. But we must also look at Fig. 1 with caution because it has been created at a specific time in the evolution of this star, using specific observational tools, specific spectral lines (typically resonance lines or other strong lines), and analysed using specific 


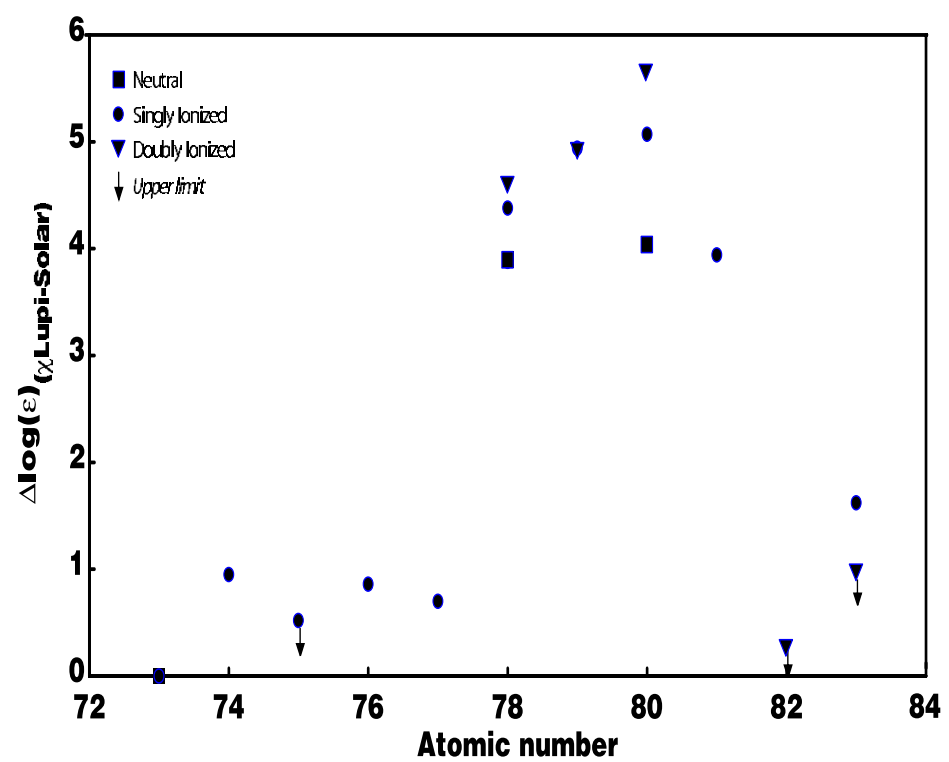

Figure 1. The $\chi$ Lupi A abundance peak for the heaviest stable elements. The singly and doubly ionized points for gold lie on top of each other. Courtesy of S. Ivarsson.

codes (ATLAS9/SYNTHE) and assumptions (LTE). Would this peak and the relationship between abundances of different ions be changed by using different spectral lines or codes that incorporate different physical assumptions (NLTE, diffusion, magnetic fields)?

Regarding the analysis of spectral line profiles in nmCP stars I would like to point out three examples of lessons learned, but warn that these lessons may easily be forgotten, or lost, if they do not become ingrained into our approach to spectrum analysis. 1) The treatment turbulence is inadequate, misunderstood, and/or incorrect. Velocity fields are clearly noticeable as asymmetric line profiles for strong lines in the spectra of Am stars (Landstreet 1998). For the hottest Am stars (for example HD 72660) the asymmetry may also be present but to only a small degree, while for the late B stars it was not noticed. The classicial LTE approach to spectrum synthesis is not capable of interpreting these asymmetries. For HgMn stars the turbulent velocity parameter has been shown to influence both the derived abundance and isotopic composition of mercury (Dolk et al. 2003). Therefore, the typical assumption of zero turbulence for late B type stars, or the use of a constant depth independent value, may lead to a misinterpretation of the spectrum. 2) The reality of ionization anomalies cannot be disputed soley by invoking the 'faulty atomic data' clause. While certain atomic data do reflect larger uncertainties than desired, it has been shown that the abundance difference for $\mathrm{Zr}$ as determined from spectral lines of Zr II and Zr III for the star $\chi$ Lupi A are larger than the uncertainties (Sikström et al. 1999). While this may seem obvious from other examples, a careful analysis of the atomic data uncertainties is typically not the concern of most astronomers, leading to claims of ionization imbalance and elemental stratification in stellar atmospheres that may not be warranted. Ionization imbalance has yet to be adequately addressed in light of the assumptions of common (LTE) model atmospheres. 3) Abundance analysis results are a by-product of the lines chosen to analyse. Lines originating from high versus low excitation states may lead to very different results. It is a common approach to determine the abundance of an element for many lines of an ion and then average the results. Since the spectral lines analysed may be taken from a large wavelength interval the chances 
are good that the lines reflect a large range in the excitation potential of their lower energy level. The use of homogeneous, LTE model atmospheres will not treat the different excitation states properly. Also, the quality of atomic data is typically not similar for high versus low excitation lines, with uncertainties increasing for higher excitation. The abundance analysis will therefore have greater uncertainties (one $\sigma$ fitting errors) when lines of mixed excitation states are used, or may produce different abundances for analyses that use only high or low excitation lines. Obvious examples of problems with the classical modeling of Fe II lines in the He-weak star 3 Cen A are presented by Wahlgren \& Hubrig (2004).

\section{Isotopic anomalies}

The composition of isotopes in stellar atmospheres provides a particularly telling account of dynamic processes at work. Observations of He-weak and HgMn stars made during the 1960s established that the elements helium, platinum, and mercury present line profiles $(\mathrm{He})$ and shifts $(\mathrm{Pt}, \mathrm{Hg}$ ) that can best be explained as variations of the isotopic composition in the region of line formation. As a result of atomic structure there was shown to be a relationship between the shift in the center of gravity of specific spectral lines of Pt II and $\mathrm{Hg}$ II with isotope composition. The q-formalism was introduced by White et al. (1976), quantifying the shift for the line $\mathrm{Hg}$ II $\lambda 3984$. The q-formalism was useful for many years and is still a useful indicator of the $\mathrm{Hg}$ isotopic composition for low to medium resolution spectroscopy. However, it has been shown that the q-formalism is untenable at high spectral resolution (Smith 1997, Wahlgren et al. 2000, Dolk et al. 2003, Woolf \& Lambert 1999). The high resolution spectra show that the heavier mercury isotopes $(\mathrm{A}=202,204)$ are prominent in most HgMn stars, in contrast to the solar system composition.

Isotopic composition may also be inferred to be different from the measurements of different spectral lines both among and between ions. From optical and HST ultraviolet spectra for the stars $\chi$ Lupi and HR 7775 the isotopic composition of platinum and mercury is suspected to be different from different ions (Wahlgren et al. 2000). The variation of isotopic composition with atmospheric depth and ionization stage is an outcome of diffusion theory (Michaud et al. 1974). Additional stars, comprising a range of atmospheric conditions need to be investigated in a manner similar to $\chi$ Lupi and HR 7775 to better serve as a constraint for diffusion.

There is also evidence for isotopic anomalies among other elements. From ultraviolet HST observations of $\chi$ Lupi are measured line shifts and synthetic spectrum fitting of the resonance line Tlin $\lambda 1908$ to be essentially only from isotope $\mathrm{A}=205$, with no contribution from $\mathrm{A}=203$. The same data set tentatively claims an anomaly for lead from a single line of $\mathrm{Pb}$ III, with the uncertainty resulting from line blending. For $\chi$ Lupi the interesting consistency among the isotopic anomalies is that the heaviest isotope is essentially the only one that is observed.

Most recently, synthetic spectrum fitting of optical lines of Ga II (Nielsen et al. 2000, Nielsen 2002) point to the distinct possibility of an anomaly for the star $\kappa$ Cnc, for which the gallium abundance is highly enhanced. Additionally, wavelength shifts measured for the CaII infrared triplet have been interpreted as an anomalous isotopic composition in several HgMn stars (Castelli \& Hubrig 2004). For both of these elements additional study is warranted to explore isotope anomalies in additional stars. But a certain caution must be interjected into the discussion. Derived isotopic compositions may depend upon the amount of turbulence assumed in the analysis and perhaps the modeling approach. These points have not been adequately documented. 


\section{Weak emission lines}

The detection of very weak emission lines in high resolution, high $\mathrm{S} / \mathrm{N}$ spectra of mid to late B type main sequence stars is an exciting discovery that imparts a little more reality into the bland thinking of B and A star atmospheres as theoretically simplistic. It is perhaps somewhat surprising that they have escaped detection until now, given that data of a necessary quality to detect them has existed for a couple of decades (post-photographic era). The few published accounts of their existence (Sigut et al. 2000, Wahlgren \& Hubrig 2000, 2001a, 2001b, 2004, Wahlgren et al. 2003) show their equivalent widths to be on the order of a few to tens of $\mathrm{m} \AA$. It is highly probable that all mid to late $\mathrm{B}$ main sequence stars create such lines, since those stars without detections are binaries with considerable flux from a secondary star that acts to dilute the visibility of the primary star emission lines. The identified lines originate from the first ionization state of mostly iron-group elements ( $\mathrm{Cr}, \mathrm{Mn}, \mathrm{Fe}, \mathrm{Ni}, \mathrm{Cu})$ as well as the elements $\mathrm{P}, \mathrm{Si}, \mathrm{Ca}$, and $\mathrm{Hg}$. The interpretation of these lines has been discussed in terms of NLTE processes, both from a traditional modeling approach (Sigut 2001a,b) and less conventional means (Wahlgren et al. 2001a, 2004). These emission lines have not been observed in the few Am stars observed for this purpose.

One interesting aspect of the MnII emission lines in HgMn star spectra is their relationship with the manganese abundance. For stars of a rather solar-like composition, often referred to as chemically normal stars, emission lines from Mn II multiplet 13 are detected. As the abundance of manganese is increased to the highest levels observed in HgMn stars, these same lines become observed as absorption lines. In cases other than the most Mn-rich or nearly solar-like Mn composition, the relative line strengths (absorption or emission) of lines in the mutiplet are not observed to be the same as the gf-values for the lines. The emission lines are quenched as the abundance increases and since the presence of certain emission lines varies with effective temperature and chemical composition we can be assured that our present atmospheric models do not properly treat the potentially stratified nature of material in the upper atmosphere.

\section{Spectrum variability}

It is generally presumed that the nmCP stars do not exhibit photometric or spectroscopic variability unless it is related to binarity. Variability is a common phenomenon among the magnetic CP stars, resulting from the inhomogeneous surface distributions of elements caused by the interaction of material with strong, global dipole magnetic fields. Photometric monitoring of $\mathrm{CP}$ stars, including nmCP stars, is being undertaken by Adelman and collaborators, but has yet to detect variability for HgMn stars.

Claims of spectroscopic variability are found in the literature but are typically not definitive. The most well known case of reported variability is for the bright, archtype HgMn star $\alpha$ And. Suspicions of spectrum variability date back to early ultraviolet satellite observations. The reality of spectrum variability for this star has only recently been realized through observations of the $\mathrm{Hg}$ II $\lambda 3984$ line (Ryabchikova et al. 1999, Ilyin 2000, Wahlgren et al. 2001, Adelman et al. 2002, Wahlgren et al. 2002). This line is the strongest optical transition of $\mathrm{Hg}$ II in $\mathrm{HgMn}$ stars and is known to display isotopic composition variations among $\mathrm{HgMn}$ stars. For $\alpha$ And this line presents undulations on a time scale of 2.38 days, the presumed rotational period. The star is also a binary, with an orbital period of 96.88 days. The application of Doppler imaging techniques to time series observations produces surface maps in the light of $\mathrm{Hg}$ II that are similar to those commonly constructed for magnetic CP stars. The $\mathrm{Hg}$ II line profile variations can thus 
be explained as an inhomogeneous surface distribution of mercury with higher concentrations occurring along the rotational equator. But can this superficial comparison between $\alpha$ And and magnetic CP stars imply that a global magnetic field exists in this HgMn star? Similar line profile variability has yet to be verified for other elements in this star, which makes one suspicious of the magnetic origins. Further observations are necessary for $\alpha$ And to extend the search for variable line profiles to other elements and wavelength regions, as well as for other $\mathrm{HgMn}$ stars to provide information regarding the parameter space for such phenomena.

\section{Magnetic fields}

The chemically peculiar stars of the upper main sequence are usually considered in terms of two separate tracks that run parallel in spectral type. They were established by the presence or absence of a magnetic field as detected spectroscopically through Zeeman broadening of magnetically sensitive spectral lines. The attributed differences to these two tracks are stark, despite the fact that the uncertainties of spectral classification for CP stars in the system of Preston (1974), which does not explicitly invoke magnetic field as a classification criterion.

One explanation for the nondetection for magnetic fields among nmCP stars is that the fields are complex, meaning that the field is not a global dipole structure, but instead is comprised of a number of magnetic dipoles or complexes that are distributed over the surface of the star. The net effect of these fields would be cancellation, resembling a solar analog. The potential for complex magnetic fields has been suggested through observations of the desaturation of magnetically sensitive line pairs in $\mathrm{HgMn}$ and Am stars. Field strengths on the order of 1 to $2 \mathrm{kG}$ were proposed for $o$ Peg (Mathys \& Lanz 1990), HD 29173 and HD 195479A (Lanz \& Mathys 1993), 74 Aqr A and $\chi$ Lupi B (Mathys \& Hubrig 1995), and for several stars by Hubrig, Castelli \& Wahlgren (1999) and Hubrig \& Castelli (2001). (The catalogue of Bychkov et al. (2003) is a useful compendium of stars with reported magnetic field measurements.) The analysis of magnetic fields in $\mathrm{nmCP}$ stars is particularly difficult due to the small values of the deviation of the equivalent width from the nonmagnetic case. Their study relies on good accuracy for the atomic data, the appropriateness of the present generation of model atmospheres, assumptions of the field structure, and high quality observational data.

The legitamacy of these results is called into question by the nondetection of any magnetic field to the detection limit of the longitudinal Zeeman effect (Shorlin et al. 2002), which measures the polarization of starlight. The absence of any such detections implies that the HgMn and Am stars do not possess field structures similar to magnetic CP stars, for which polarization measurements are providing modelers with additional constraints on the magnetic geometry. The polarization measurements are not yet conclusive for $n m C P$ stars because they have not been made for the same targets for which claims of magnetic fields have been made. But even in the eventual absence of any support for magnetic fields from either Zeeman line broadening or polarization we should address the question of whether it is possible for there to be other evidences indicative of a magnetic field. Is it possible that a magnetic field (global dipole or not) be found at sufficient depth below the photosphere so that it is not detected by the aforementioned techniques, but manifests itself in some other manner, such as an enhancement of spectral lines of a particularly sensitive ion? Is attributing line strength enhancements to diffusion processes alone masking the presence of a magnetic field? It is difficult to believe that nmCP stars have no magnetic fields, when magnetic fields are thought to permeate the astrophysics of star and galaxy formation. 


\section{Stratification}

Over the past decade the concept of vertical stratification of abundances in stellar atmospheres has been brought to the test by observations. In terms of interpreting CP star atmospheres by diffusion theory it is quite sensible to think that some (or all) elements may not be distributed homogeneously throughout the line formation region. In the presence of vertical stratification the abundance of an element may be different if determined from lines of different ionization stages or from lines originating from considerably different excitation potentials. In addition, the isotopic composition of an element may also vary with depth. Much work has been undertaken on stratification among the magnetic CP stars and the rapidly oscillating Ap stars (roAp). For the nmCP stars stratification has been suggested from observations involving i) ionization anomalies of $\mathrm{Ga}$ (Smith 1995), ii) abundance trends of $\mathrm{Cr}$ II lines in the wing of $\mathrm{H} \beta$ (Savanov \& Kochukhov 1998, Savanov \& Hubrig 2003), iii) He I line profiles (Dworetsky, this volume), and iv) weak emission lines (Sigut 2001a,b).

The arguements in favor of stratification from each of these techniques are weak. In all cases it is the modeling of the stellar atmospheres that is the most speculative aspect of the problem. What can be said is that the plane-parallel homogeneous model atmosphere does not serve to reproduce spectral observations in some cases. Another contention is the role of turbulence in the modeling. A depth independent turbulent velocity is the normal approach to modeling line profiles and equivalent widths. But this may not be a reasonable assumption, since turbulence profiles can lead to equivalent width variations.

Regarding the ionization anomaly for gallium, the case has been made for stratification by using high resolution optical spectra for higher excitation lines with $I U E$ spectra used for resonance lines. The latter suffers from severe line blending (Nielsen 2002 and paper in preparation), which when accounted for reduces the magnitude of the anomaly. Mixing data of different quality requires caution in the claims of the results.

Ionization anomalies do exist, as noted above, when the spectrum is analysed with LTE computer codes. The application of NLTE codes is progressing but at a rather slow pace, in part due to a need for appropriate model atoms (particularly, collisional cross-sections) but also the result of momentum among astronomers to fend off changing their habits regarding the use of LTE codes. Claims of stratification from observations of ionization imbalance must for the present be tempered if the analysis was conducted in LTE.

\section{Crossing boundaries}

The study of CP stars has benefitted from earlier efforts to bring order to the perplexing variety of peculiarities exhibited by the stars of the upper main sequence. Through spectral classification we are able to identify common peculiarity traits and promote the study of certain groupings of stars in more depth. However, at some point the continuance of compartmentized studies alone becomes unhealthy because they are not equipped to answer questions of a more general nature, those questions posed regarding origins and evolution. The literature on the observations of CP stars is dominated by studies of specific stellar groups and does not promote thinking in terms of continuity across longer baselines of effective temperature, mass, or magnetic field properties.

Recent work is beginning to address connecting the nmCP star groupings to explain observed changes in spectral peculiarities over spectral type. Wahlgren \& Dolk (1998) initiated a program to study chemical composition trends across the boundary separating the HgMn from the hot-Am stars near spectral type A0. It has since been realized that the abundances of certain elements (lanthanides) have a more continuous behavior 
than previously thought while other elements (Pt-group) display a rather discontinuous behavior across this boundary. Dolk (2002) undertook to extract from the literature on $\mathrm{nmCP}$ stars the abundance trends of 27 elements over the effective temperature range 7000 to 14000 K. From this one sees that the spread in abundance is large $(>0.5$ dex) for most elements across the entire temperature range. This spread reflects both starto-star variations and the systematics of different data sets and their analysis. Observed trends include deficiencies (He, C, N, O) or enhancements (Nd, Pr, Ce, Ba, Sr) at all temperatures as well as a tendency for abundance to increase (Mn, P, Ca, Sc, Ti, Y, Zr) or decrease (Ni, Al, S) with increasing temperature. These trends now serve as generalized constraints for theory, since the influence of stellar age is not taken into account.

The evolution of spectrum peculiarity in the vicinity of spectral type A0 has been taken up by Adelman et al. (2003). They have proposed that the hot-Am stars (spectral type A0-A2) have evolved from the stars at the cool end of the HgMn class. The mass range of interest is between 2.5 and 3.0 solar masses, where both HgMn and hot-Am star classes have members. This is an interesting idea that requires further theoretical consideration. Although not addressed by this group, an additional consideration should be whether isotopic anomalies and their temporal evolution are consistent with this suggestion. The known isotopic composition of mercury for the HgMn stars in this mass range display no trend with effective temperature or luminosity.

Much more work remains to be done before understanding the relationship between $\mathrm{HgMn}$ and hot-Am stars. Likewise, the relationships between the He-weak and HgMn stars as well as the hot-Am stars with the classical Am stars need to be explored. Still other boundaries remain to be crossed. The distinction between magnetic and nonmagnetic stars may be all too artificial and need to be readdressed in terms of continuity. And there may even be psychological barriers that must be crossed before agreement can be reached on the implementation of improved analysis tools. The many interesting problems posed by CP stars can best be solved by the expertise of many disciplines and their resolve to cooperate.

\section{Acknowledgements}

It is a pleasure to acknowledge financial support received from the Swedish Academy of Sciences.

\section{References}

Adelman, S.J., Adelman, A.S., \& Pintado, O.I. 2003, A 6 A 397, 267

Adelman, S.J., Cowley, C. R., Leckrone, D. S., Roby. S. W., Wahlgren, G. M. 1993, ApJ 419, 276

Adelman, S.J., Gulliver, A. F., Kochukhov, O. P., Ryabchikova, T. A. 2002, ApJ 575, 449

Adelman, S.J., Proffitt, C. R., Wahlgren, G. M., Leckrone, D. S., Dolk, L. 2004, ApJS in press

Burkhart, C. \& Coupry, M. F. 1997, A\& A 318, 870

Bychkov, V.D., Bychkova, L. V. \& Madej, J. 2003, A\&A 407, 631

Castelli, F. \& Hubrig, S. 2004, AछA 421, L1

Dolk, L. 2002, PhD Thesis, Lund Obervatory, Lund University

Dolk, L., Wahlgren, G.M. \& Hubrig, S. 2003, A\&A 402, 299

Evans, N.R. 1995, ApJ 445, 393

Evans, N.R., Vinko, J. \& Wahlgren, G.M. 2000, AJ 120, 407

Evans, N.R., Carpenter, K., Rbinson, R. et al. 1999, ApJ 524, 379

Farthmann, M. et al. 1994, A\&A 291, 919

Hubrig, S. \& Castelli, F. 2001, A\&A 375, 963

Hubrig, S. \& Mathys, G. 1995, Comments Astrophys. 18, 167 
Hubrig, S., Castelli, F. \& Wahlgren, G.M. 1999, A\&\&A 346, 139

Hui-Bon-Hoa, A., Burkhart, C. \& Alecian, G. 1997, A\&A 323, 901

Hui-Bon-Hoa, A. \& Alecian, G. 1998, A\&A 332, 224

Iliev, I. Kh., Fenovcik, M., Budaj, J., et al. 2005, These Proceedings, 301

Ilyin, I.V. 2000, PhD Thesis, Oulu University

Ivarsson, S. et al. 2004, $A \mathscr{E A}$ in press

Kurucz, R.L. 1995, CD-ROM 23

Landstreet, J. 1998, A\&A 338, 1041

Lanz, T. \& Mathys, G. 1993, A\&A 280, 486

Leckrone, D.S., Proffitt, C. R., Wahlgren, G. M., et al. 1999, AJ 117, 1454

Mathys, G. \& Hubrig, S. 1995, A\&A 293, 810

Mathys, G. \& Lanz, T. 1990, A\& A 230, L21

Monier, R. \& Richard, O. 2005, These Proceedings, AP2

Michaud, G., Reeves, H. \& Charland, Y. 1974, A\& $A$ 37, 313

Nielsen, K., Karlsson, H. \& Wahlgren, G.M. 2000, A\& $A$ 363, 815

Nielsen, K. 2002, PhD Thesis, Lund Observatory, Lund University

Preston, G.W. 1974, ARA\&A 12, 257

Ryabchikova, T. 1998, Contr. Astron. Obs. Skalnaté Pleso 27, 319

Ryabchikova, T. A., Malanushenko, V. P., \& Adelman, S. J. 1999, A\&A A 351, 963

Savanov, I.S. 1998, Astronomy Reports 42, 508

Savanov, I. \& Hubrig, S. 2003, A\& $A$ 410, 299

Savanov, I.S. \& Kochukhov, O.P. 1998, AstL 24, 516

Shorlin, S.L.S., Wade, G. A., Donati, J.-F. et al. 2002, A\&\&A 392, 637

Sigut, T.A.A., Landstreet, J. D., Shorlin, S. L. S. 2000, ApJ 530, L89

Sigut, T.A.A. 2001a, ApJ 546, L115

Sigut, T.A.A. 2001b, $A \& A$ A 377, L27

Sikström, C.-M. et al. 1999, A\&A 343, 297

Smith, K.C. 1995, A\&A 297, 237

Smith, K.C. 1996, Ap\&SSS 237, 77

Smith, K.C. 1997, A\&\& 319,928

Stelzer, B., Huélamo, N., Hubrig, S. et al. 2003, A\&A 407, 1067

Wahlgren, G.M. \& Dolk, L. 1998, Contr. Astron. Obs. Skalnaté Pleso 27, 314

Wahlgren, G.M. \& Evans, N.R. 1998, A\&AA 332, L33

Wahlgren, G.M. et al. 1993, in: M.M. Dworetsky, F. Castelli \& R. Faraggiana (eds.), Peculiar Versus Normal Phenomena in A-Type Stars, IAU Coll. No. 138, ASP Conf. Ser. 44, 121

Wahlgren, G.M., Dolk, L., Kalus, G. et al. 2000, ApJ 539, 908

Wahlgren, G.M. \& Hubrig, S. 2000, A\& A 362, L13

Wahlgren, G.M. \& Hubrig, S. 2001a, in: G. Mathys, S.K. Solanki \& D.T. Wickramasinghe (eds.), ASP Conf. Ser. 248, p. 369

Wahlgren, G.M. \& Hubrig, S. 2001b, ASP Conf. Ser. 248, p.365

Wahlgren, G.M. \& Hubrig, S. 2004, A\&A 418, 1073

Wahlgren, G.M., Ilyin, I. \& Kochukhov, O. 2001, BAAS 33, 1506

Wahlgren, G.M., et al. 2002, in: K.G. Strassmeier \& A. Washuettl, 1st Potsdam Thinkshop on Sunspots \& Starspots, poster proceedings, (Astrophysical Institute Potsdam), p. 87

Wahlgren, G.M., Hubrig, S. \& Ivarsson, S. 2003, in: L.A. Balona, H.F. Henrichs \& R. Medupe (eds.), International Conference on Magnetic Fields in $O, B$, and A Stars ASP Conf. Ser. 305 , p. 256

White, R.E., Vaughan, A. H., Jr., Preston, G. W., \& Swings, J. P. 1976, ApJ 204, 131

Woolf, V.M. \& Lambert, D.L. 1999, ApJ 521, 414 